TITLE:

Increased risk of disease progression in younger men: Analysis of factors predicting biochemical failure and castration-resistant prostate cancer after high-dose intensity-modulated radiation therapy for nonmetastatic prostate cancer( Dissertati ...

$\operatorname{AUTHOR}(S):$

Aizawa, Rihito

\title{
CITATION:
}

Aizawa, Rihito. Increased risk of disease progression in younger men: Analysis of factors predicting biochemical failure and castration-resistant prostate cancer after high-dose intensity-modulated radiation therapy for nonmetastatic prostate ca...

ISSUE DATE:

2021-03-23

URL:

https://doi.org/10.14989/doctor.k23081

RIGHT: 


\title{
Clinical-Prostate cancer
}

\section{Increased risk of disease progression in younger men: Analysis of factors predicting biochemical failure and castration-resistant prostate cancer after high-dose intensity-modulated radiation therapy for nonmetastatic prostate cancer}

\author{
Rihito Aizawa, M.D. ${ }^{\text {a }}$ Kenji Takayama, M.D. ${ }^{\text {a,c }}$, Kiyonao Nakamura, M.D., Ph.D. ${ }^{\text {, }}$ \\ Takahiro Inoue, M.D., Ph.D. ${ }^{\mathrm{b}, \mathrm{d}}$, Toshinari Yamasaki, M.D., Ph.D. ${ }^{\mathrm{b}}$, \\ Takashi Kobayashi, M.D., Ph.D. ${ }^{\text {, }}$, Shusuke Akamatsu, M.D., Ph.D. ${ }^{\text {b }}$, \\ Osamu Ogawa, M.D., Ph.D. ${ }^{\mathrm{b}}$, Takashi Mizowaki, M.D., Ph.D. ${ }^{\mathrm{a}}{ }^{*}$ \\ ${ }^{a}$ Department of Radiation Oncology and Image-applied Therapy, Graduate School of Medicine, Kyoto University, 54 Shogoin Kawahara-cho, \\ Sakyo-ku, Kyoto 606-8507 Japan \\ ${ }^{\mathrm{b}}$ Department of Urology, Graduate School of Medicine, Kyoto University, Sakyo-ku, Kyoto Japan \\ ${ }^{\mathrm{c}}$ Department of Radiology, Tenri Hospital, Tenri-shi, Nara, Japan \\ ${ }^{\mathrm{d}}$ Department of Nephro-Urologic Surgery and Andrology, Mie University Graduate School of Medicine, Tsu, Mie, Japan
}

Received 8 May 2020; received in revised form 21 August 2020; accepted 26 September 2020

\begin{abstract}
Background: The aim of this study was to investigate the clinical significance of the effect of age on disease control in men who received high-dose intensity-modulated radiation therapy (IMRT) for nonmetastatic prostate cancer (NMPCa).

Methods: NMPCa patients with favorable intermediate to very high-risk features (National Comprehensive Cancer Network risk classification) treated with IMRT at our institution between September 2000 and May 2011 were analyzed retrospectively. Treatment consisted of high-dose IMRT (74-78 Gy/37-39 fractions) combined with 6 months of neoadjuvant hormonal therapy. Multivariable analysis using Fine and Gray's regression model was performed to evaluate whether age at initiation of IMRT was associated with biochemical failure (BF) and castration-resistant prostate cancer (CRPC) progression.

Results: A total of 367 patients were analyzed. The median follow-up period was 8.8 years after IMRT. The 5- and 10-year BF rates were 22.1 and $31.7 \%$, and those of CRPC rates were 4.5 and $12.6 \%$, respectively. Multivariable analysis revealed that a younger age (cutoff: 70 years old) at the initiation of IMRT was significantly correlated with both a higher BF rate (hazard ratio: $1.691, P=0.0064)$ and higher CRPC rate (hazard ratio: $2.579, P=0.0079$ ).

Conclusions: Younger men with NMPCa had increased risks of BF and CRPC after high-dose IMRT, and may benefit from more intensive treatments. Our findings should be further tested in prospective studies. (c) 2020 Elsevier Inc. All rights reserved.
\end{abstract}

Keywords: Biochemical failure; Castration-resistant prostate cancer; Intensity-modulated radiation therapy; Predictive factor; Prostate cancer; Younger men

\section{Introduction}

Definitive external-beam radiotherapy (EBRT) combined with hormonal therapy (HT) has been established as a standard

\footnotetext{
*Corresponding author. Tel.: +81-75-751-3762; fax: +81-75-771-9749

E-mail address: mizo@kuhp.kyoto-u.ac.jp (T. Mizowaki).
}

of practice for nonmetastatic prostate cancer (PCa) [1]. With advances in mechanical engineering and computer technology, intensity-modulated radiation therapy (IMRT) enables a safe increase in the radiation dose by selectively protecting the rectum, facilitating its widespread clinical use.

Meanwhile, the association between a younger age and tumor aggressiveness has been reported in several types of 
tumor, such as breast cancer [2,3]. However, regarding PCa, the clinical impact of age on disease control remains controversial [4,5]. Although several studies have reported a markedly lower rate of disease control and poorer survival outcomes in younger PCa patients [6-10], the studies on definitive EBRT for nonmetastatic PCa (NMPCa) have been based mainly on the use of 70-Gy or lower doses via 3-dimensional conformal radiotherapy (3D-CRT) [6,7], which is suboptimal to estimate clinical outcomes in the current IMRT era. In addition, those studies described the results of EBRT monotherapy or EBRT with various durations of combined HT. Hence, the impact of age on disease control under conditions of modern high-dose irradiation combined with uniform HT remains unknown. Specifically, if the risk of disease failure significantly increases in younger patients, then the age at treatment initiation may act as a novel predictive factor that is useful to identify the subgroup potentially benefitting the most from an increased treatment intensity.

Therefore, the objective of the current study was to evaluate the impact of age on biochemical failure (BF) and castration-resistant prostate cancer (CRPC) progression in men who received high-dose IMRT and HT for NMPCa, using a retrospective cohort of a single institution with a long follow-up period (median; 8.8 years) and a large number of patients $(n=367)$.

\section{Materials and methods}

This study followed the tenets of the Helsinki Declaration, with approval from the institutional ethical review board (approval number: R1048). Written informed consent was obtained from all patients.

\subsection{Patients}

We retrospectively reviewed our prospectively maintained institutional PCa database, and searched for eligible patients. The eligibility criteria were as follows: (1) NMPCa categorized into favorable or unfavorable intermediate-risk (IR), high-risk (HR), or very high-risk (VHR) groups according to the National Comprehensive Cancer Network risk classification ver. 2. 2019 [1] with histological confirmation of adenocarcinoma; (2) treated with IMRT to the prostate and seminal vesicles (SVs) alone between September 2000 and May 2011 at our institution; (3) duration of neoadjuvant HT (NA-HT) $<12$ months; and (4) prescription dose $\geq 74$ Gy. Patients with CRPC at the initiation of IMRT were excluded.

\subsection{Neoadjuvant hormonal therapy and intensity- modulated radiation therapy}

We previously reported the details of our institutional treatment protocol [11-13]. In brief, treatments consisted of short-term NA-HT and high-dose IMRT. NA-HT comprised 6 months of combined androgen blockage. However, regarding the duration and contents, there were minor variations because a large number of patients were introduced to our hospital after HT had been initiated, and patients with liver dysfunction or special requests were administered the luteinizing hormone-releasing hormone analogue alone. In the current study, patients exceptionally treated with long-term NA-HT (cut-off: 1 year) were excluded, as described in the eligibility criteria. For IMRT, a total of $78 \mathrm{~Gy}$ in 39 fractions was prescribed for the prostate and SVs (the base, proximal two-thirds, or whole of SVs according to the risk), which was reduced to $74 \mathrm{~Gy}$ in IR PCa with $\mathrm{T} 1-2 \mathrm{~b}$ disease. In addition, the total was reduced by 4 to $12 \mathrm{~Gy}$ in patients with risk factors for rectal bleeding (cases whereby the total dose was reduced to lower than 74 Gy were excluded from this analysis). Prophylactic pelvic nodal irradiation was not performed.

\subsection{Patient follow-up and salvage therapy}

The follow-up schedule was previously described [12,13]. No adjuvant therapy, including adjuvant HT (A-HT), was performed because we designed the treatment protocol before the establishment of the combination of long-term AHT for unfavorable PCa under the condition of high-dose irradiation as the standard of care. Instead, we initiated salvage therapy in an early phase after recurrence (prostate-specific antigen $[\mathrm{PSA}]>4.0 \mathrm{ng} / \mathrm{mL}$ ). Salvage therapy basically comprised continuous/intermittent combined androgen blockage, luteinizing hormone-releasing hormone analogue monotherapy, or antiandrogenic agent monotherapy. For patients who developed oligo-metastasis to pelvic lymph nodes following IMRT, salvage EBRT to the upper pelvis in combination with long-term HT was applied [14]. No androgen receptor axis targeted (ARAT) agent or chemotherapy was used in a castration-sensitive setting.

\subsection{Statistical analyses}

The timing of occurrence of each event was calculated from the date of IMRT initiation. BF was evaluated based on the Phoenix definition [15]. CRPC was defined as the earliest timing of the following: (1) PSA increase of $25 \%$ from the nadir and a minimum $2.0 \mathrm{ng} / \mathrm{mL}$ under castration levels of testosterone $(<50 \mathrm{ng} / \mathrm{mL})$ tested at a minimum of 1-week intervals (the definition of the Prostate Cancer Working Group 3 [PCWG3]) [16], or during HT (if testosterone levels were not assessed with appropriate timing); (2) change in the contents of salvage HT due to PSA elevation, locoregional progression, or development of distant metastasis; or (3) locoregional progression or development of distant metastasis during salvage therapy. PSA elevation during the off-period of intermittent HT was not counted as a CRPC event. The rates of BF and CRPC were estimated using the cumulative incidence method, accounting for death without each event as a competing risk.

The patients were divided into two age groups by the median age (70 years old), and Chi-square analysis or 
Student's $t$ test was used to compare pretreatment characteristics, follow-up periods, and number of other causes of death without $\mathrm{BF}$ between the age groups. To evaluate the clinical impact of age on BF and CRPC progression, univariate analysis (UVA) and multivariable analysis (MVA) were conducted using Fine and Gray's regression model. Along with age at the initiation of IMRT ( $\leq 70 \mathrm{vs.} \geq 71$ years old), pretreatment PSA ( $>20$ vs. $\leq 20 \mathrm{ng} / \mathrm{mL}$ ), clinical T stage (T3-4 vs. T1-2), Gleason Score (GS) sum ( $\geq 8$ vs. $\leq 7$ ), and number of cores with a GS sum of 8 to 10 ( $\geq 5$ vs. $\leq 4)$, were included as covariates.

A $P$ value $<0.05$ denoted significance. All statistical analyses were performed using $\mathrm{R}$ version 3.1.1 (The $\mathrm{R}$ Foundation for Statistical Computing, Vienna, Austria).

\section{Results}

\subsection{Patient characteristics}

A total of 373 patients met the eligibility criteria. Among them, pelvic lymph node surgical dissection before IMRT was performed in one, bone or pelvic lymph node metastasis before IMRT was retrospectively detected in 3, and AHT was irregularly added in an affiliated hospital due to the doctor's or patient's request in 2. Therefore, these 6 patients were excluded, and the remaining 367 patients were included in the analysis.

The median patient age was 70 (interquartile range [IQR]: 65-75) years old at IMRT initiation. The median pretreatment PSA level was 16.2 (IQR: $9.7-30.7) \mathrm{ng} / \mathrm{mL}$. Approximately $40 \%$ of the patients $(n=148)$ showed a GS of $\geq 8$, and more than half of the patients $(n=192)$ had $\geq$ T3a disease. Subsequently, 24, 66, 191, and 86 patients were categorized into favorable IR, unfavorable IR, HR, and VHR groups, respectively [1]. The characteristics of the 367 patients are summarized in Table 1. The patient characteristics stratified by age at the initiation of IMRT ( $\leq 70$ vs. $\geq 71$ years old) are presented in Table 2 . No significant difference regarding initial clinicopathological features was observed between the age groups.

\subsection{Neoadjuvant hormonal therapy and intensity- modulated radiation therapy}

All patients were treated with NA-HT for a median duration of 6.4 (IQR: 5.0-7.8) months. For IMRT, a total dose of 78 or 74 Gy was prescribed to $226(61.6 \%)$ and 141 (38.4\%) patients, respectively The details of treatments are summarized in Table 1.

\subsection{Oncological outcomes and association with age}

The median follow-up period for the entire cohort was 8.8 (IQR: 6.9-10.8) years. No significant difference in follow-up periods was observed between the age groups (median: 9.0 years for $\leq 70$ years old vs. 8.6 years for
Table 1

Patient and treatment characteristics

\begin{tabular}{|c|c|}
\hline \multicolumn{2}{|l|}{ Age (years) } \\
\hline Median & 70 \\
\hline IQR & $65-75$ \\
\hline \multicolumn{2}{|l|}{ Clinical T stage, $\mathrm{n}(\%)$} \\
\hline $\mathrm{T} 1 \mathrm{c}$ & $52(14.2)$ \\
\hline $\mathrm{T} 2 \mathrm{a}$ & $53(14.4)$ \\
\hline $\mathrm{T} 2 \mathrm{~b}$ & $35(9.5)$ \\
\hline $\mathrm{T} 2 \mathrm{c}$ & $35(9.5)$ \\
\hline T3a & $138(37.6)$ \\
\hline $\mathrm{T} 3 \mathrm{~b}$ & $49(13.4)$ \\
\hline $\mathrm{T} 4$ & $5(1.4)$ \\
\hline \multicolumn{2}{|l|}{ iPSA (ng/mL) } \\
\hline Median & 16.2 \\
\hline IQR & $9.7-30.7$ \\
\hline \multicolumn{2}{|l|}{ Gleason score, n (\%) } \\
\hline 6 & $31(8.4)$ \\
\hline 7 & $185(50.4)$ \\
\hline 8 & $89(24.3)$ \\
\hline 9 & $57(15.5)$ \\
\hline 10 & $5(1.4)$ \\
\hline \multicolumn{2}{|l|}{$\mathrm{NCCN}$ risk classification, $\mathrm{n}(\%)$} \\
\hline Favorable intermediate-risk & $24(6.5)$ \\
\hline Unfavorable intermediate-risk & $66(18.0)$ \\
\hline High-risk & $191(52.1)$ \\
\hline Very high-risk & $86(23.4)$ \\
\hline \multicolumn{2}{|l|}{ Duration of NA-HT (months) } \\
\hline Median & 6.4 \\
\hline IQR & $5.0-7.8$ \\
\hline \multicolumn{2}{|l|}{ IMRT dose, $\mathrm{n}(\%)$} \\
\hline 78 Gy & $226(61.6)$ \\
\hline 74 Gy & $141(38.4)$ \\
\hline \multicolumn{2}{|l|}{ Nadir PSA (ng/mL) } \\
\hline Median & 0.019 \\
\hline IQR & $0.008-0.077$ \\
\hline
\end{tabular}

Abbreviations: IMRT $=$ intensity-modulated radiation therapy; iPSA = pretreatment prostate-specific antigen; IQR = interquartile range; NA-HT = neoadjuvant hormonal therapy; $\mathrm{NCCN}=$ the National Comprehensive Cancer Network risk classification ver. 2. 2019; PSA = prostate-specific antigen.

$\geq 71$ years old, $P=0.079$; Table 2 ). There were 49 deaths, of which 28 patients died from other causes without BF. During the follow-up, 110 patients developed disease failure with a median period of 3.3 (IQR: 1.8-5.4) years after IMRT, and BF was the initial failure pattern in all of them. Among them, salvage HT was initiated in 95 patients due to continuous PSA elevation or clinical failure, and 39 patients progressed to CRPC with a median period of 5.8 (IQR: 3.9 -7.9) years after IMRT. The 5- and 10-year BF rates were $22.1 \%$ (95\% confidence interval [CI]: 18.0-26.6) and $31.7 \%$ (95\% CI: 26.5-36.9), and the 5- and 10-year CRPC rates were $4.5 \%$ (95\% CI: $2.7-7.0$ ) and $12.6 \%$ (95\% CI: 8.9-16.8), respectively (Fig. 1). Those rates stratified by the National Comprehensive Cancer Network risk classification are illustrated in Fig. 2A, B. In the UVA, a younger age was significantly correlated with a higher incidence of both $\mathrm{BF}(P=0.02)$ and $\mathrm{CRPC}(P=0.012)$. The differences in the cumulative incidence of BF and CRPC between age groups ( $\leq 70$ vs. $\geq 71$ years old) are illustrated in Fig. 3A, B. 
Table 2

Pre- and post-treatment characteristics by age ( $\leq 70 \mathrm{vs.} \geq 71$ years old)

\begin{tabular}{|c|c|c|c|}
\hline & \multicolumn{2}{|c|}{ Group } & \multirow[b]{2}{*}{$p$ value } \\
\hline & Age $\leq 70$ & Age $\geq 71$ & \\
\hline No. of patients & 189 & 178 & \\
\hline Follow-up (years) & & & 0.079 \\
\hline Median (IQR) & $9.0(7.0-10.9)$ & $8.6(6.7-10.7)$ & \\
\hline $\begin{array}{l}\text { Clinical T stage, } \mathrm{n} \\
(\%)\end{array}$ & & & 0.813 \\
\hline $\mathrm{T} 1$ & $29(15.4)$ & $23(12.9)$ & \\
\hline $\mathrm{T} 2$ & $65(34.4)$ & 58 (32.6) & \\
\hline T3a & $67(35.4)$ & $71(39.9)$ & \\
\hline $\mathrm{T} 3 \mathrm{~b}-4$ & $28(14.8)$ & $26(14.6)$ & \\
\hline Combined GS, n (\%) & & & 0.616 \\
\hline 6 & $14(7.4)$ & $17(9.6)$ & \\
\hline 7 & $101(53.4)$ & $84(47.2)$ & \\
\hline 8 & $45(23.8)$ & $44(24.7)$ & \\
\hline $9-10$ & $29(15.3)$ & $33(18.5)$ & \\
\hline iPSA (ng/mL) & & & 0.575 \\
\hline Median (IQR) & $15.0(9.6-25.1)$ & $18.0(9.9-33.0)$ & \\
\hline $\begin{array}{l}\text { cores with a combined } \\
\text { GS of } 8-10\end{array}$ & & & 0.888 \\
\hline$\leq 4$ & $168(88.9)$ & $160(89.9)$ & \\
\hline$\geq 5$ & $21(11.1)$ & $18(10.1)$ & \\
\hline $\begin{array}{l}\text { NCCN risk } \\
\text { classification, } \mathrm{n}(\%)\end{array}$ & & & 0.13 \\
\hline $\begin{array}{l}\text { Favorable } \\
\text { intermediate-risk }\end{array}$ & $13(6.9)$ & $11(6.2)$ & \\
\hline $\begin{array}{l}\text { Unfavorable } \\
\text { intermediate-risk }\end{array}$ & $41(21.7)$ & $25(14.0)$ & \\
\hline High-risk & $88(46.5)$ & $103(57.9)$ & \\
\hline Very high-risk & 47 (24.9) & 39 (21.9) & \\
\hline $\begin{array}{l}\text { Duration of NA-HT } \\
\text { (months) }\end{array}$ & & & 0.362 \\
\hline Median (IQR) & $6.5(5.3-7.9)$ & $6.2(4.8-7.8)$ & \\
\hline IMRT dose, n (\%) & & & 0.504 \\
\hline 78Gy & $120(63.5)$ & $106(59.5)$ & \\
\hline 74Gy & $69(36.5)$ & $72(40.5)$ & \\
\hline Nadir PSA (ng/mL) & & & 0.525 \\
\hline Median (IQR) & $0.02(0.01-0.07)$ & $0.02(0.01-0.08)$ & \\
\hline $\begin{array}{c}\text { Other cause of death } \\
\text { without } \mathrm{BF}, \mathrm{n}(\%)\end{array}$ & $6(3.2)$ & $22(12.4)$ & 0.00183 \\
\hline
\end{tabular}

Abbreviations: GS = Gleason score; IMRT = intensity-modulated radiation therapy; $\mathrm{iPSA}=$ pretreatment prostate-specific antigen;

$\mathrm{IQR}=$ interquartile range; NA-HT = neoadjuvant hormonal therapy; $\mathrm{NCCN}=$ the National Comprehensive Cancer Network risk classification ver. 2. 2019; $\mathrm{PSA}=$ prostate-specific antigen; $\mathrm{BF}$, biochemical failure.

Specifically, the 5- and 10-year BF rates were 27.4\% (95\% CI: $21.1-33.9)$ and $37.7 \%$ (95\% CI: 30.1-45.3) for younger patients ( $\leq 70$ years old), but $16.4 \%$ (95\% CI: $11.3-22.4)$ and $25.0 \%$ (95\% CI: $18.4-32.0)$ for older patients ( $\geq 71$ years old), respectively (Fig. 3A). The 5- and 10-year CRPC rates were $5.9 \%$ (95\% CI: 3.1-9.9) and 17.8\% (95\% CI: 11.9 -24.7 ) for younger patients ( $\leq 70$ years old), but $2.9 \%$ (95\% CI: $1.1-6.4)$ and $6.8 \%$ (95\% CI: 3.4-11.8) for older patients ( $\geq 71$ years old), respectively (Fig. 3B). In the MVA, a younger age remained an independent predictive factor for $\mathrm{BF}$ (hazard ratio [HR] 1.691, 95\% CI: 1.159-2.466, $P=0.0064$ ) and CRPC progression (HR: $2.579,95 \%$ CI: $1.282-5.187$,

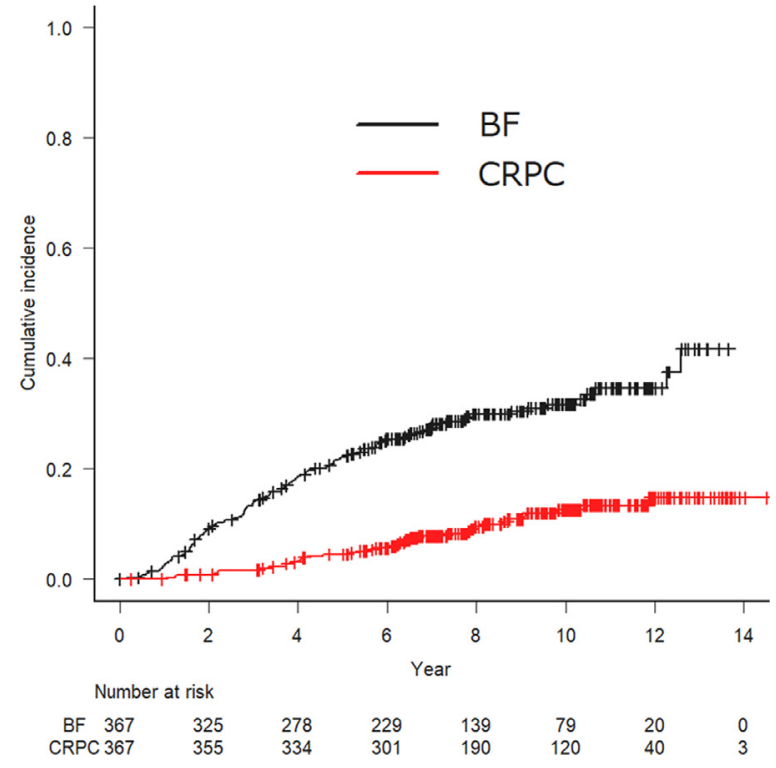

Fig. 1. Cumulative incidence curves of biochemical failure and castrationresistant prostate cancer among the entire cohort.Abbreviations: $\mathrm{BF}=$ biochemical failure; $\mathrm{CRPC}=$ castration-resistant prostate cancer.

$P=0.0079)$ with adjustment for other covariates. Details of the results of UVA and MVA are summarized in Table 3.

\section{Discussion}

In the current study of 367 patients with NMPCa who were treated with high-dose IMRT, a significant correlation was observed between a younger age and disease progression after adjusting for other known predictive factors. Of note, we evaluated factors predicting CRPC progression, which is considered a reasonable surrogate for PCa-specific mortality (PCSM) [17]. This may be of marked value in that it is considered difficult to analyze predictive factors affecting PCSM in a direct manner because of greatly improved survival outcomes reported recently. To our knowledge, studies investigating factors predicting CRPC progression after definitive EBRT for NMPCa are very limited [18].

As the results of UVA, in which our patient cohort was divided into two groups at the median age (70 years old), a $12.7 \%$ increase in the $\mathrm{BF}$ rate at 10 years was observed for $\leq 70$-year-old men compared with $\geq 71$-year-old men (BF rate at 10 years: 37.7 vs. $25.0 \%$, respectively, $P=0.02$ ). This trend was reproduced in MVA with adjustment for other covariates (HR: 1.691, 95\% CI: 1.159-2.466, $P=0.0064$ ). Similar to our results, according to a metaanalysis of 5 prospective studies of definitive EBRT for NMPCa (median dose: 70 Gy), a younger age (cut-off: 70 years old) was an independent predictive factor for both metastasis (HR: $0.72,95 \%$ CI: $0.63-0.83, P<0.0001$ ) and PCSM (HR: $0.78,95 \%$ CI: $0.66-0.92, P<0.0001$ ) [6]. We hypothesize that early testosterone recovery (TR) in younger men is one possible explanation for this age-dependent 
(a)

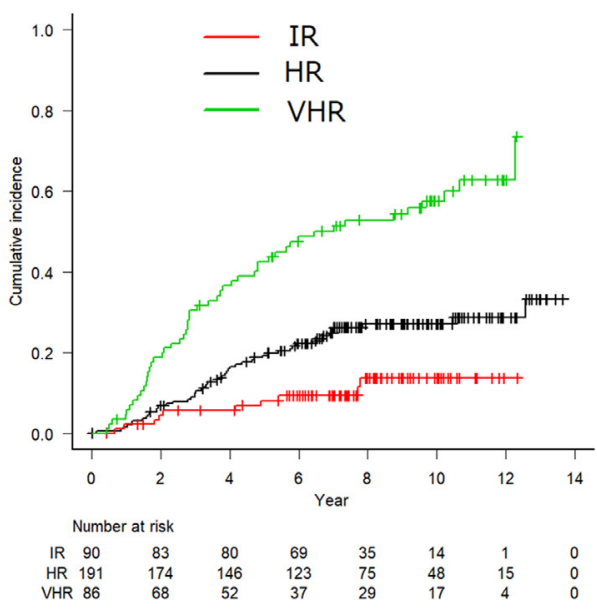

(b)

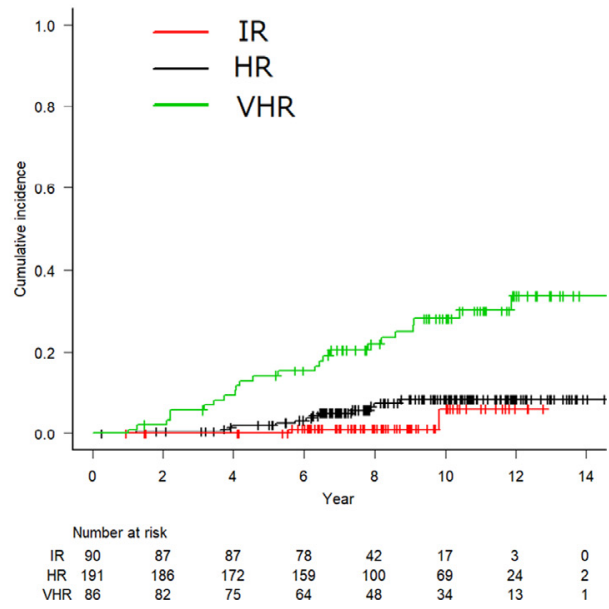

Fig. 2. Cumulative incidence curves of biochemical failure among each risk group (A), and castration-resistant prostate cancer among each risk group (B) according to the National Comprehensive Cancer Network risk classification ver. 2. 2019. Abbreviations: BF = biochemical failure; $C R P C=$ castration-resistant prostate cancer; $\mathrm{HR}=$ high-risk; IR = intermediate-risk (favorable and unfavorable); VHR = very high-risk.

(a)

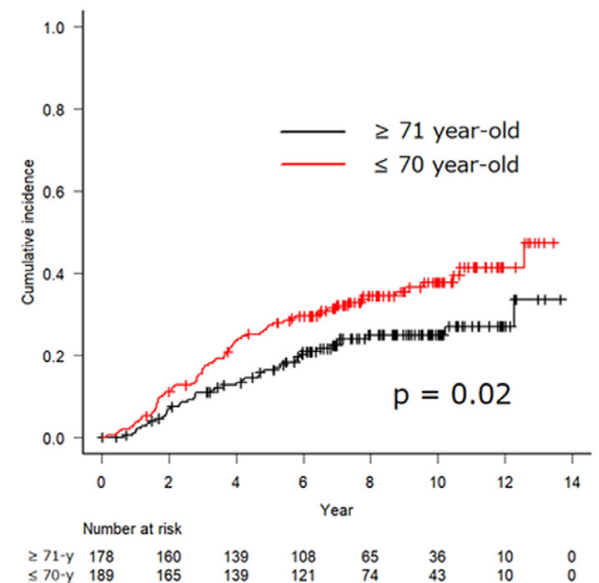

(b)

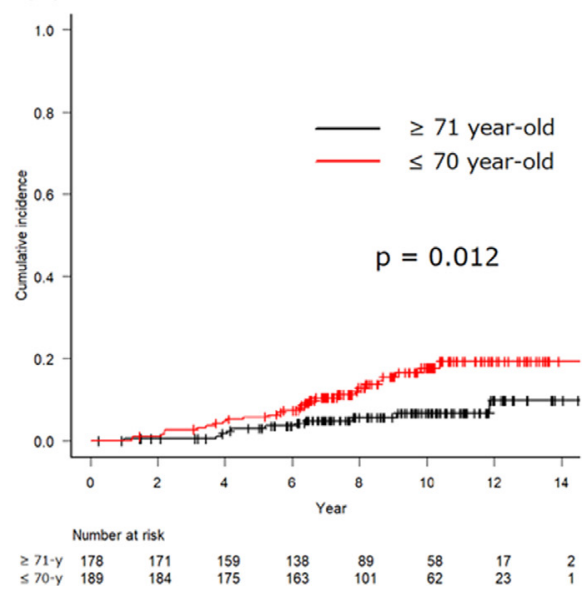

Fig. 3. Cumulative incidence curves of biochemical failure (A), and castration-resistant prostate cancer (B) stratified by the age at the initiation of intensitymodulated radiation therapy ( $\leq 70 \mathrm{vs.} \geq 71$ years old).

Table 3

Univariate and multivariable analyses of risk factors for biochemical failure and castration-resistant prostate cancer

\begin{tabular}{|c|c|c|c|c|c|c|}
\hline \multirow[t]{2}{*}{ Factor } & \multicolumn{3}{|c|}{ Univariate analysis } & \multicolumn{3}{|c|}{ Multivariable analysis } \\
\hline & HR & $95 \% \mathrm{CI}$ & $P$ & HR & $95 \% \mathrm{CI}$ & $P$ \\
\hline \multicolumn{7}{|l|}{ Biochemical failure } \\
\hline iPSA: $>20 \mathrm{ng} / \mathrm{mL}$ vs. $\leq 20 \mathrm{ng} / \mathrm{mL}$ & 3.435 & $2.319-5.089$ & $<0.001$ & 2.628 & $1.655-4.174$ & $<0.001$ \\
\hline clinical T stage: T3-4 vs. T1-2 & 2.28 & $1.513-3.437$ & $<0.001$ & 1.371 & $0.8659-2.171$ & 0.18 \\
\hline GS sum: $\geq 8$ vs. $\leq 7$ & 2.079 & $1.427-3.027$ & $<0.001$ & 1.5 & $0.9567-2.352$ & 0.077 \\
\hline cores with GS sum $8-10 ; \geq 5$ vs. $\leq 4$ & 3.755 & $2.528-5.578$ & $<0.001$ & 1.749 & $1.016-3.012$ & 0.044 \\
\hline Age: $\leq 70-y$ vs. $\geq 71-y$ & 1.575 & $1.074-2.309$ & 0.02 & 1.691 & $1.159-2.466$ & 0.0064 \\
\hline \multicolumn{7}{|l|}{ Castration-resistant prostate cancer } \\
\hline iPSA: $>20 \mathrm{ng} / \mathrm{mL}$ vs. $\leq 20 \mathrm{ng} / \mathrm{mL}$ & 2.373 & $1.238-4.545$ & 0.0092 & 1.355 & $0.6107-3.008$ & 0.45 \\
\hline clinical T stage: $\mathrm{T} 3-4$ vs. $\mathrm{T} 1-2$ & 4.488 & $1.869-10.78$ & $<0.001$ & 3.654 & $1.437-9.294$ & 0.0065 \\
\hline GS sum: $\geq 8$ vs. $\leq 7$ & 2.378 & $1.25-4.525$ & 0.0083 & 1.822 & $0.8742-3.797$ & 0.11 \\
\hline cores with GS sum $8-10 ; \geq 5$ vs. $\leq 4$ & 3.316 & $1.669-6.592$ & $<0.001$ & 1.39 & $0.546-3.54$ & 0.49 \\
\hline Age: $\leq 70-y$ vs. $\geq 71-y$ & 2.43 & $1.211-4.875$ & 0.012 & 2.579 & $1.282-5.187$ & 0.0079 \\
\hline
\end{tabular}

Abbreviations: GS = Gleason Score; HR = hazard ratio; iPSA = pretreatment prostate-specific antigen; $95 \% \mathrm{CI}=95 \%$ confidence interval. 
difference in BF observed in our cohort. It has been reported that time to TR increases with advancing age [19,20], and this delayed TR after EBRT among older populations may result in a lower BF rate. Indeed, increased time to TR was reportedly associated with a decreased risk of PCSM (HR: 0.89, 95\% CI: 0.82-0.96, $P=0.003$ ) among NMPCa patients treated with EBRT and HT for 6 months [21]. Because our patients did not receive A-HT following the completion of IMRT, their timing of initial failure after IMRT may have been directly affected by TR. In this regard, the current standard long-term A-HT is considered to be reasonable especially for younger men in order to suppress TR.

More importantly, this study revealed that the younger patients had a significantly higher CRPC rate than the older patients (CRPC rate at 10 years: 17.8 vs. $6.8 \%$, respectively, $P=0.012$ ). This phenomenon cannot be explained solely by the early TR in younger patients. A difference in biological backgrounds between early-onset $\mathrm{PCa}$ and elderly-onset PCa patients was recently reported, and inherited mutations in DNA damage repair genes (BRCA mutations) comprise one type of reported factor associated with an aggressive clinical course among young patients [22]. However, given the low prevalence of BRCA mutations (less than 5.0\%) and the much younger age at onset (usually younger than 50 years old) [23], such an association does not sufficiently explain the marked difference observed in CRPC rates. Therefore, it is difficult to provide a reasonable explanation for this phenomenon. There might exist unknown biological factors contributing to this age-dependency of tumor aggressiveness. Our results suggest the survival benefit of increasing the treatment intensity for younger patients. Recently, the up-front use of ARAT agents or docetaxel for hormone-sensitive metastatic PCa patients yielded better oncologic outcomes in several randomized controlled trials [24]. Therefore, although somewhat speculative, the use of those agents in a neoadjuvant setting or in first-line salvage therapy may be a promising method for younger PCa patients. Our findings should be further investigated, especially in the setting of prospective trials.

We acknowledge that an excess of non-PCa-specific mortality (NPCSM) and presumably shorter follow-up periods in the older group may have led to an underestimation of the likelihood of BF and CRPC progression. However, our follow-up periods of the older group were considered long enough to estimate late occurrence of each event, and no significant difference in follow-up periods was observed between the age groups $(P=0.079)$. Furthermore, the separation of cumulative incidence curves between the age groups was observed from the beginning, where these effects are considered to be minimal (Fig. 3). Therefore, the cause of the higher rates of $\mathrm{BF}$ and $\mathrm{CRPC}$ progression observed in the younger group could not be traced back to a difference in NPCSM.
The current study had several limitations, including its retrospective nature. Testosterone levels were not routinely evaluated among the patients who did not develop disease progression due to a restriction of the national insurance system, making it difficult to directly investigate the correlation between testosterone levels and age. Furthermore, the patients included in this study received prostate-only IMRT and short-term NA-HT alone because we designed the treatment protocol before the establishment of the combination of prophylactic pelvic nodal irradiation or longterm A-HT for unfavorable PCa as the standard of care. Our results may not be directly applicable to patients treated with the current standard of care, because prostateonly IMRT and short-term NA-HT alone are considered as suboptimal treatment for HR and VHR PCa compared with the current standard. Therefore, our findings are not conclusive but merely hypothetical. However, our data may consequently provide a more accurate observation of the direct correlation between pretreatment factors and the risk of disease progression without being masked by A-HT. Therefore, we believe that our results provide baseline data to understand the age-dependency of the tumor aggressiveness of PCa. Given the growing demand for evidence to support individualized treatments, these findings would be of particular importance.

\section{Conclusions}

This study showed that a younger age was significantly correlated with higher BF and CRPC rates among NMPCa patients treated with high-dose IMRT. We consider that younger patients would benefit from more intensive treatments, such as the up-front use of ARAT agents or docetaxel in a neoadjuvant setting or in first-line salvage treatment. Further investigation is warranted to confirm our findings.

\section{Conflicts of interest}

The authors made no disclosures.

\section{Acknowledgments}

This work was partly supported by JSPS KAKENHI Grant Number JP16K10390. We thank Dr. Ryo Ashida for his assistance with statistics and data-checking of the analysis.

\section{References}

[1] National Comprehensive Cancer network. NCCN Guidelines; prostate cancer version 2.2019. The category of prostate cancer; https:// wwwnccnorg/professionals/physician_gls/defaultaspx\#site. Accessed April 172019

[2] Ono Y, Yoshimura M, Hirata K, Yamauchi C, Toi M, Suzuki E, et al. The impact of age on the risk of ipsilateral breast tumor recurrence after breast-conserving therapy in breast cancer patients with a 
$>5 \mathrm{~mm}$ margin treated without boost irradiation. Radiat Oncol 2019; $14: 121$

[3] Jones HA, Antonini N, Hart AA, Peterse JL, Horiot JC, Collin F, et al. Impact of pathological characteristics on local relapse after breast-conserving therapy: a subgroup analysis of the EORTC boost versus no boost trial. J Clin Oncol 2009:27:4939-47.

[4] Bernard B, Burnett C, Sweeney CJ, Rider JR, Sridhar SS. Impact of age at diagnosis of de novo metastatic prostate cancer on survival. Cancer 2020;126:986-93.

[5] Salinas CA, Tsodikov A, Ishak-Howard M, Cooney KA. Prostate cancer in young men: an important clinical entity. Nat Rev Urol 2014;11:317-23.

[6] Hamstra DA, Bae K, Pilepich MV, Hanks GE, Grignon DJ, McGowan DG, et al. Older age predicts decreased metastasis and prostate cancer-specific death for men treated with radiation therapy: metaanalysis of radiation therapy oncology group trials. Int J Radiat Oncol Biol Phys 2011;81:1293-301.

[7] Proust-Lima C, Taylor JM, Williams SG, Ankerst DP, Liu N, Kestin LL, et al. Determinants of change in prostate-specific antigen over time and its association with recurrence after external beam radiation therapy for prostate cancer in five large cohorts. Int J Radiat Oncol Biol Phys 2008;72:782-91.

[8] Hong SK, Nam JS, Na W, Oh JJ, Yoon CY, Jeong CW, et al. Younger patients have poorer biochemical outcome after radical prostatectomy in high-risk prostate cancer. Asian J Androl 2011;13:719-23.

[9] Inoue T, Kinoshita H, Terada N, Kobayashi T, Yamasaki T, Matsui $\mathrm{Y}$, et al. Evaluation of prognostic factors after radical prostatectomy in pT3b prostate cancer patients in Japanese population. Jpn J Clin Oncol 2015;45:780-4.

[10] Kimura T, Onozawa M, Miyazaki J, Matsuoka T, Joraku A, Kawai K, et al. Prognostic impact of young age on stage IV prostate cancer treated with primary androgen deprivation therapy. Int $\mathrm{J}$ Urol 2014;21:578-83.

[11] Norihisa Y, Mizowaki T, Takayama K, Miyabe Y, Matsugi K, Matsuo Y, et al. Detailed dosimetric evaluation of intensity-modulated radiation therapy plans created for stage $\mathrm{C}$ prostate cancer based on a planning protocol. Int J Clin Oncol 2012;17:505-11.

[12] Aizawa R, Takayama K, Nakamura K, Inoue T, Yamasaki T, Kobayashi $\mathrm{T}$, et al. Ten-year outcomes of high-dose intensity-modulated radiation therapy for nonmetastatic prostate cancer with unfavorable risk: early initiation of salvage therapy may replace long-term adjuvant androgen deprivation. Int J Clin Oncol 2019;24:1247-55.

[13] Aizawa R, Takayama K, Nakamura K, Inoue T, Yamasaki T, Kobayashi $\mathrm{T}$, et al. Low incidence of late recurrence in patients with intermediate-risk prostate cancer treated by intensity-modulated radiation therapy plus short-term androgen deprivation therapy. Int J Clin Oncol 2020;25:713-9.
[14] Sato GE, Aizawa R, Nakamura K, Takayama K, Inoue T, Yamasaki $\mathrm{T}$, et al. Long-term clinical outcomes of salvage pelvic radiation therapy for oligo-recurrent pelvic lymph nodes after definitive externalbeam radiation therapy for non-metastatic prostate cancer. J Radiat Res 2020;61:622-8.

[15] Roach 3rd M, Hanks G, Thames H Jr, Schellhammer P, Shipley WU, Sokol GH, et al. Defining biochemical failure following radiotherapy with or without hormonal therapy in men with clinically localized prostate cancer: recommendations of the RTOGASTRO Phoenix Consensus Conference. Int J Radiat Oncol Biol Phys 2006;65:965-74.

[16] Scher HI, Morris MJ, Stadler WM, Higano C, Basch E, Fizazi K, et al. Trial design and objectives for castration-resistant prostate cancer: updated recommendations from the prostate cancer clinical trials working group 3. J Clin Oncol 2016;34:1402-18.

[17] Hussain M, Goldman B, Tangen C, Higano CS, Petrylak DP, Wilding $\mathrm{G}$, et al. Prostate-specific antigen progression predicts overall survival in patients with metastatic prostate cancer: data from Southwest Oncology Group Trials 9346 (Intergroup Study 0162) and 9916. J Clin Oncol 2009;27:2450-6.

[18] Spratt DE, Zumsteg ZS, Pei X, Romesser PB, Yamada J, Kollmeier MA, et al. Predictors of castration-resistant prostate cancer after dose-escalated external beam radiotherapy. Prostate 2015; 75:175-82.

[19] D’Amico AV, Chen MH, Renshaw AA, Loffredo M, Kantoff PW. Interval to testosterone recovery after hormonal therapy for prostate cancer and risk of death. Int J Radiat Oncol Biol Phys 2009;75:10-5.

[20] Tsumura H, Satoh T, Ishiyama H, Hirano S, Tabata K, Kurosaka S, et al. Recovery of serum testosterone following neoadjuvant and adjuvant androgen deprivation therapy in men treated with prostate brachytherapy. World J Radiol 2015;7:494-500.

[21] D’Amico AV, Renshaw AA, Loffredo B, Chen MH. Duration of testosterone suppression and the risk of death from prostate cancer in men treated using radiation and 6 months of hormone therapy. Cancer 2007;110:1723-8.

[22] Castro E, Goh C, Olmos D, Saunders E, Leongamornlert D, Tymrakiewicz M, et al. Germline BRCA mutations are associated with higher risk of nodal involvement, distant metastasis, and poor survival outcomes in prostate cancer. J Clin Oncol 2013;31:1748-57.

[23] Leongamornlert D, Mahmud N, Tymrakiewicz M, Saunders E, Dadaev T, Castro E, et al. Germline BRCA1 mutations increase prostate cancer risk. Br J Cancer 2012;106:1697-701.

[24] Wallis CJD, Klaassen Z, Bhindi B, Goldberg H, Chandrasekar T, Farrell AM, et al. Comparison of abiraterone acetate and docetaxel with androgen deprivation therapy in high-risk and metastatic hormonenaive prostate cancer: a systematic review and network meta-analysis. Eur Urol 2018;73:834-44. 